\title{
ESTUDO DA RELAÇÃO ENTRE ATIVIDADE FÍSICA E 0 CICLO DO SONO/VIGÍLIA
}

\author{
Virvalene Costa de Melo*1, Ellyton Valente Saraiva ${ }^{2}$, Jeanderson Barros Pinheiro ${ }^{2}$, Smayk \\ Barbosa Sousa ${ }^{3}$, Moisés Simão Santa Rosa de Souza3, Antonio César Matias de Lima ${ }^{3}$, Tamirez \\ Santana Muniz ${ }^{3}$, José Roberto Zaffalon Jr. ${ }^{4}$, Rômulo Teixeira dos Santos ${ }^{5}$ \\ and Gileno Edu Lameira Melo ${ }^{3}$
}

\begin{abstract}
${ }^{1}$ Departamento de Morfologia e Ciências Fisiológicas da Universidade do Estado do Pará (UEPA); ${ }^{2}$ Licenciado em Educação Física pela Universidade do Estado do Pará (UEPA); ${ }^{3}$ Departamento de Desporto da Universidade do Estado do Pará (UEPA); ${ }^{4}$ Departamento de Ginástica e Artes Corporais da Universidade do Estado do Pará (UEPA); ${ }^{5}$ Residente em Saúde Mental (UEPA/FHCGV)
\end{abstract}

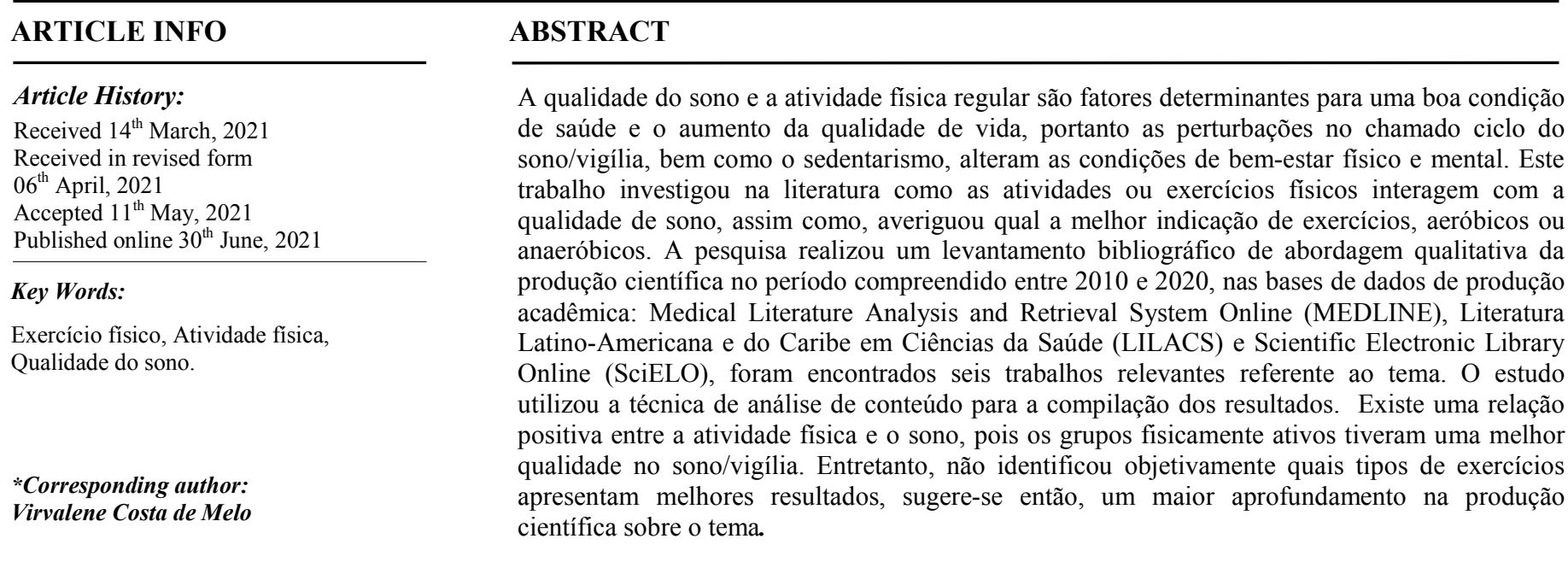

Copyright @ 2021, Janaína Gaia Ribeiro Dias et al. This is an open access article distributed under the Creative Commons Attribution License, which permits unrestricted use, distribution, and reproduction in any medium, provided the original work is properly cited.

Citation: Virvalene Costa de Melo, Ellyton Valente Saraiva, Jeanderson Barros Pinheiro, Smayk Barbosa Sousa, Moisés Simão Santa Rosa de Souza, Antonio César Matias de Lima, Tamirez Santana Muniz, José Roberto Zaffalon Jr., Rômulo Teixeira dos Santos and Gileno Edu Lameira Melo. 2021. "Color perception in children with sicklecell anemia and potential impacts in cognitive development", International Journal of Development Research, 11, (06), 48004-48007.

\section{INTRODUÇÃO}

O sono é classificado, como um processo biológico complexo, com o passar do tempo, alterna-se com o estado de sono-vigília e é mediado por processos hormonais e neurais (McCarley, 2007; Carter PJ et al, 2011; Luyster et al apud Zanuto, 2014). Tais processos fisiológicos alteram a temperatura corporal, a função cardíaca e a produção de hormônios, levando a um estado neurológico restaurador, importante para o crescimento (infância e adolescência), aprendizagem / memória e o funcionamento do corpo. Para Buela (1990) o sono é um estado funcional, reversível e cíclico, com alguns comportamentos característicos como uma imobilidade relativa e o aumento do limite de respostas aos estímulos externos. Em um contexto orgânico, acontecem variações dos parâmetros biológicos, juntamente com uma modificação da atividade mental, que se refere ao comportamento de dormir.
A percepção da qualidade do sono é descrita segundo Ropke (2017) como dificuldade em adormecer e dificuldade em manter o sono, acordar cedo, como a pessoa se sente após adormecer e como a pessoa se sente sobre a qualidade do sono. Podem reduzir a qualidade do sono, mudanças e fatores psicológicos, sociais e ambientais, como: uso de drogas; hábitos de fumar; consumo de bebidas alcoólicas; bebidas com cafeína. Pereira et al. (2018) esclarecem sobre a quantidade de sono necessária, que embora seja preciso dormir algumas horas a cada 24 horas, não é a quantidade e sim a qualidade do sono obtido nessas horas, associada à necessidade individual, que determinam a normalidade desta função. Ou seja, é preciso não somente dormir muito, mas sim, ter uma boa qualidade de sono. As causas mais comuns de prejuízo do sono é a insônia, que pode ser resultado da demanda dos afazeres diários, uso de medicamentos, fatores pessoais e estilos de vida. No que se trata da fragmentação do sono, esta resulta em um sono de quantidade e qualidade inadequadas, sendo consequência de condições médicas e/ou fatores 
Tabela 1. Combinação dos termos da pesquisa

\begin{tabular}{|l|l|l|}
\hline Palavra & Operador booleano & Palavra \\
\hline Exercício & And & Sono \\
\hline Atividade física & And & Qualidade do sono \\
\hline Exercício & And & Índice de qualidade do sono \\
\hline
\end{tabular}

Tabela 2. Relação dos trabalhos com programa de treinamento

\begin{tabular}{|c|c|c|c|}
\hline Autor & Tipo de atividade envolvida & Programa de treinamento & Avaliação da qualidade de sono \\
\hline Pereira et al. (2018) & Aeróbica & $\begin{array}{l}8 \text { semanas de caminhada em intensidade moderada, } \\
\text { sendo } 3 \text { sessões de } 40 \text { minutos por semana }\end{array}$ & $\begin{array}{l}\text { Houve uma melhora de } 4,9 \text { no escore do } \\
\text { PSQI no grupo que participou das sessões } \\
\text { de treinamento }\end{array}$ \\
\hline Santiago et al. (2015) & Anaeróbica & $\begin{array}{l}12 \text { semanas de sessão de treinos, com uma sessão a } \\
\text { cada } 4 \text { semanas, totalizando } 3 \text { sessões }\end{array}$ & $\begin{array}{l}100 \% \text { dos participantes que treinaram pela } \\
\text { manhã evoluíram a qualidade de sono de } \\
\text { ruim para boa }\end{array}$ \\
\hline Steffens et al.(2011) & Aeróbica & $\begin{array}{l}32 \text { sessões de caminhada, sendo realizadas } 2 \text { vezes } \\
\text { por semana com duração de } 60 \text { minutos( } 15 \text { minutos } \\
\text { de alongamento, } 30 \text { min caminhada e } 15 \text { min de } \\
\text { relaxamento) }\end{array}$ & $\begin{array}{l}\text { Houve uma melhora na média de } \\
\text { qualidade do sono dos participantes de } \\
1,72 \text { pontos no PSQI }\end{array}$ \\
\hline
\end{tabular}

Fonte: Autor

ambientais que o interrompem. Como consequências da alteração do padrão de sono podem ocorrer reduções da eficiência do processamento cognitivo, do tempo de reação e responsividade atencional, além de déficit de memória, aumento da irritabilidade, alterações metabólicas, endócrinas e quadros hipertensivos (MELLO et al., 2005). Conforme trata Avidan (2017), a ansiedade e os distúrbios do sono são problemas comuns na maioria da população e, são estabelecidos motivos comuns para consulta na atenção primária, o que influencia na qualidade de vida e função pessoal dos indivíduos. A Insônia se caracteriza como um distúrbio generalizado, e que é normalmente crônico, afetando $5 \%$ a $15 \%$ da população total o que se relaciona a uma grande redução na qualidade de vida. Normalmente os transtornos de ansiedade estão também associados com a insônia, sendo um problema comum na população afetando significativamente a qualidade de vida e a disposição do indivíduo. Parafraseando Geib et al. (2003), outros autores afirmam que, a linha divisória entre a normalidade e o distúrbio pode ser estabelecida por meio de criteriosa avaliação diagnóstica, a qual necessariamente precede e orienta a conduta terapêutica. A maioria dos distúrbios de sono são clinicamente importantes e tratáveis com medidas nãofarmacológicas, que incluem a orientação sobre rotinas e rituais de sono, atividades de vida diária e condições ambientais. Segundo estudos, por volta de $20 \%$ a $40 \%$ da população mundial possui algum problema relacionado ao sedentarismo ou a qualidade de sono, podendo apresentar sintomas depressivos ou de ansiedade, aumento do risco de acidentes cardiovasculares, queda no rendimento de trabalho, entre outros transtornos que venha a ser adquiridos com a má qualidade de sono (MELLO et al., 2005). Sendo que no Brasil, por volta de dez a vinte milhões de pessoas apresentam problemas com relação ao sono (LINS-FILHO et al.,2019), esse é um dado bastante alarmante, visto que a qualidade do sono está diretamente relacionada a doenças crônicas, o que ocasiona em uma má qualidade de vida. $\mathrm{O}$ exercício físico se tornou nos últimos anos, fundamental para a promoção da saúde, assim como, no aumento da qualidade de vida, o que ajuda na diminuição dos riscos de desenvolvimento de doenças crônicas e crescimento da longevidade. Por outro lado, a perturbação do ciclo sono/vigília tem como resultado, consequências significativas para a saúde e bem-estar (MARTINS et al.,2001). Os exercícios são reconhecidos pela organização American Sleep Disorders Association como uma intervenção não-farmacológica para a melhoria do padrão de sono. Porém, efetivamente, a prescrição de exercícios físicos com essa finalidade ainda é reduzida, possivelmente como um reflexo da falta de conhecimento por parte de professores e médicos dos benefícios dos exercícios nessa área (MARTINS et al., 2001). O presente estudo teve por objetivo investigar na literatura como as atividades ou exercícios físicos interagem com a qualidade de sono, assim como, averiguar qual exercícios, aeróbicos ou anaeróbicos se mostram positivo sobre o ciclo do sono/vigília.

\section{METODOLOGIA}

Foi adotado como procedimento metodológico de revisão bibliográfica proposta por Gil (2008), de cunho qualitativo. A produção acadêmica foi buscada nas bases de dados da Medical Literature Analysis and Retrieval System Online (MEDLINE) Literatura Latino-Americana e do Caribe em Ciências da Saúde (LILACS) e Scientific Electronic Library Online (SciELO); no período compreendido entre 2010 e 2020. Além da restrição temporal, também foram filtrados os artigos encontrados em duplicação, título, resumo, periódicos em Língua Portuguesa, usando como operador booleano o "and", combinando os termos utilizados para que cada resultado da pesquisa contenha todos os termos. Segue, na Tabela 01 , a combinação dos termos utilizados para a consulta nos bancos de dados, que ainda foram filtradas por título, resumo e assunto.

\section{RESULTADOS E DISCUSSÃO}

Na pesquisa foram encontrados 260 trabalhos, sendo que 127 na base de dados do LILACS, 116 na base de dados do SciELO, e 17 na base de dados do MEDLINE. Foram excluídos os que não relacionavam a atividade física com a qualidade do sono, restando 53 artigos. Feita a leitura dos resumos dos 53 artigos, foram selecionados 11 para leitura na íntegra. Após esse procedimento, foram selecionados 6 trabalhos, levando em consideração se na sua avaliação foi utilizado o Índice de Qualidade de Sono de Pittsburgh, qual a intervenção quanto ao tipo de atividade física e a conclusão deles. A pesquisa de Steffens et al. (2011), submeteu sua amostra a aplicação do questionário de Índice de Qualidade de Sono de Pittsburg (PSQI) antes e depois do programa de treinamento, com intensidade entre $60 \%$ e $75 \%$ da frequência cardíaca máxima estimada. Essa população era constituída por 9 mulheres com idade média de 48 anos, portadoras da síndrome da fibromialgia. Os resultados mostram melhora no humor, com resultados médios de: tensão (de 9,78 para 6,22), depressão (de 9,56 para 6,88 ), raiva (de 7,33 para 3,66 ) e confusão mental (de 8,56 para $5,11)$. Esse estudo demonstra uma evolução geral na qualidade de vida dessa população, por meio de treinamento aeróbico, em especial na qualidade do sono, que apresentou resultado expressivamente positivo, passando de uma média pré-teste de 11,29 para 9,57 pósteste. O estudo de Santiago et al. (2015) teve um objetivo de intervenção com caráter exploratório, pois realizava uma sessão de treino de força a cada quatro semanas, onde os participantes as realizavam pela manhã, tarde ou noite, e no dia seguinte respondiam o PSQI, ou seja, avaliou o efeito de uma sessão de treinamento resistido sobre a qualidade do sono. Sua amostra era composta por seis estudantes do IFPE - Campus Vitória de Santo Antão, PE, Brasil. 
O protocolo de treinamento era composto por oito exercícios: cadeira extensora, cadeira flexora, panturrilha e cadeira abdutora, remada baixa, rosca direta, tríceps pulley e elevação lateral. Todos com três séries de oito repetições com $60 \%$ da carga para uma repetição máxima, e intervalos entre 60 e 90 segundos entre cada série. Os resultados apontaram que o turno de treinamento anaeróbico matutino foi o que melhor refletiu em melhoria na qualidade do sono dos indivíduos, enquantoo treinamento noturno se mostrava ineficaz para alguns, quanto a esta variável.

Já o estudo de Pereira etal. (2018) contou com uma amostra de 16 estudantes saudáveis, com faixa etária entre 18 e 37 anos, da Universidade Federal Rural de Pernambuco (UFRPE), Campus Recife, Pernambuco, Brasil. Onde 8 faziam parte do grupo treinado (realizaram testes ergométricos) e 8 não foram submetidos a nenhuma intervenção. Todos os participantes responderam ao PSQI antes e após as 8 semanas. O grupo treinado realizava as sessões de testes de esforço máximo em esteira (Protocolo de Ellestad), de intensidade moderada com frequência cardíaca máxima entre 55-65\%. Este grupo mostrou expressiva melhora na pontuação do PSQI, passando de uma média de 6,8 para 3,7 pontos no referido questionário após o protocolo de treinamento; enquanto o grupo de sedentários não apresentou melhoras com relação à qualidade do sono. Mostrando assim, que um protocolo orientado de treinamento aeróbico pode melhorar a qualidade do sono. Na Tabela 02 se encontra a relação dos trabalhos com os programas de treinamento. Dentre os resultados das buscas realizadas neste trabalho também foram encontrados artigos que não se utilizaram de intervenção por meio de treinamento. Sua abordagem se deu pela aplicação de questionários, incluindo o PSQI, de acordo com seus objetivos, como mostrado na Tabela 03. (idade média de $52,4 \pm 1,08$ ) e 20 eram sedentárias (idade média de $49,25 \pm 0,97)$. Após a aplicação do PSQI, observou que, quanto a qualidade do sono, o grupo que praticava musculação:25\%apresentaram classificação boa, $65 \%$ ruim e $10 \%$ distúrbio do sono; enquanto, das praticantes de caminhada: $15 \%$ apresentaram boa qualidade do sono, $40 \%$ ruim e $45 \%$ distúrbio do sono. Já nas mulheres sedentárias, o cenário é pior, apenas $5 \%$ apresentaram boa qualidade de sono, $60 \%$ ruim e $35 \%$ mostraram distúrbio do sono. Percebe-se que, de uma amostra de 40 mulheres ativas apenas 8 apresentaram qualidade do sono geral boa, enquanto 21 apresentaram qualidade de sono ruim e 19 distúrbio do sono. Entretanto, para as mulheres sedentárias os resultados se mostraram piores, com 1 indivíduo com boa qualidade de sono, $12 \mathrm{com}$ qualidade ruim e 7 com distúrbio no ciclo do sono. Vale ressaltar que, em geral, nessa pesquisa, o exercício aeróbico mostrou melhores resultados quanto à qualidade do sono. O estudo de Silva et al. (2018) com 385 idosos, sendo 126 do sexo masculino e 259 do sexo feminino, verificou a relação entre sintomas de depressão e a presença e intensidade da dor crônica com a qualidade do sono e o nível de atividade física, sendo que 58,2\% apresentaram quadro de dor crônica e $41,8 \%$ não. Quanto à qualidade de sono: $43,4 \%$ apresentaram qualidade de sono boa, $50,4 \%$ qualidade de sono ruim e $6,2 \%$ apresentaram distúrbios de sono. No que se refere aos sintomas depressivos: $69,4 \%$ não os apresentaram, enquanto $30,6 \%$ apresentaram tais sintomas. Ao correlacionar essas variáveis, Silva et al. (2018) constataram que os idosos que apresentavam dor crônica tinham 3,36 vezes mais chances de ocorrência de sintomas de depressão, e que existe uma correlação moderada entre os sintomas de depressão e qualidade do sono, sendo que os idosos que apresentaram sintomas depressivos possuíam piores escores de

Tabela 3. Relação dos trabalhos que não realizaram programa de treinamento

\begin{tabular}{|c|c|c|}
\hline Autor (ano) & Objetivo & $\begin{array}{l}\text { Materiais utilizados } \\
\text { (Questionários) }\end{array}$ \\
\hline Campos et al. (2011) & $\begin{array}{l}\text { Identificar o nível de atividade física e sua relação com sonolência } \\
\text { excessiva diurna qualidade de sono em } 15 \text { mulheres com fibromialgia, } \\
\text { com idade média de } 58 \pm 7 \text { anos. }\end{array}$ & $\begin{array}{l}\text { - Questionário Internacional de Atividade Física (IPAQ); } \\
\text { - Índice de Qualidade de sono de Pittsburgh (PSQI); } \\
\text { - Escala de Sonolência de Epworth (ESE) }\end{array}$ \\
\hline Portela et al. (2017) & $\begin{array}{l}\text { Analisar a qualidade do sono em mulheres climatéricas ativas, } \\
\text { praticantes de musculação ou caminhada, e sedentárias. }\end{array}$ & • Índice de Qualidade de sono de Pittsburgh (PSQI); \\
\hline Silva et al. (2018) & $\begin{array}{l}\text { Verificara relação entre sintomas depressivos e a presença e intensidade } \\
\text { da dor crônica com a qualidade do sono e o nível de atividade física. }\end{array}$ & $\begin{array}{l}\text { - Em seguida, a escala visual numérica de dor (EVN); } \\
\text { - Questionário Internacional de Atividade Física (IPAQ) } \\
\text { - GeriatricDepressionScale(GDS) }\end{array}$ \\
\hline
\end{tabular}

Fonte: Autor

Em estudo transversal com questionários, Campos et al. (2011), relacionou a qualidade geral do sono com o nível de atividade física em 15 mulheres com fibromialgia, com idade entre 45 e 75 anos. Dessas, 10 eram minimamente ativas e 5 inativas fisicamente. Após a aplicação dos questionários de nível de atividade física, índice de qualidade de sono e escala de sonolência, os resultados apontaram que 5 mulheres do grupo minimamente ativo e 2 do grupo inativo apresentaram distúrbios do sono; enquanto 4 mulheres do primeiro grupo e 3 do segundo apresentaram qualidade do sono ruim, ou seja, mais de $90 \%$ da amostra apresentou qualidade de sono ruim ou distúrbios no ciclo do sono, e apenas 1 pessoa apresentou boa qualidade de sono. Vale ressaltar que na comparação entre o índice de atividade física (IPAQ) e sonolência diurna excessiva (Escala de sonolência de Epworth - ESE), 60\% total da amostra, mulheres minimamente ativas e inativas, apresentou pontuação classificada como sonolência diurna excessiva. Dos resultados encontrados por Campos et al. (2011), 46,7\% das participantes da pesquisa apresentaram qualidade de sono ruim e 46,7\% apresentaram distúrbios de sono. Também demonstra que todas as pacientes apresentaram baixo do nível de atividade física, sendo que 33,3\% apresentaram-se inativas e $66,7 \%$ minimamente ativas. Pode-se constatar que mulheres com fibromialgia, mesmo que minimamente ativas, apresentam um ciclo de sono ruim, o que pode estar relacionado ao quadro clínico desta população. Portela et al. (2017), em investigação sobre o índice de qualidade do sono de 60 mulheres climatéricas (estado fisiológico do hipoestrogenismo, com a interrupção definitiva dos ciclos menstruais), onde, 20 praticavam

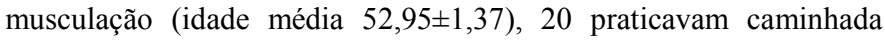

qualidade de sono(50,4\% classificados com sono ruim). Verificou-se também a relação entre o nível de atividade física e os sintomas de depressão, onde: os idosos insuficientemente ativos são os que apresentavam maior prevalência de sintomas de depressão $(49,92 \%)$, ao ponto que os idosos sem esses sintomas $(58,1 \%)$ eram ativos ou muito ativos. Por se tratar de um estudo com outras variáveis, existiu uma correlação entre elas, mostrando que os idosos com maior presença ou intensidade de dor crônica, sintomas de depressão e com pouca atividade física apresentaram um índice geral de qualidade de sono ruim ou distúrbios de sono. Um bom nível de atividade física se mostrou um fator essencial para a qualidade de vida desses indivíduos, pois quando correlacionadas com as outras, evidenciou que quanto menor ela for mais irá propiciar a presença das outras.

\section{CONCLUSÃO}

Observou-se uma correlação positiva entre o exercício físico e qualidade do sono, ou seja, grupos populacionais que são mais ativos tendem a apresentar melhor qualidade do sono, bem como os indivíduos que possuem distúrbios relacionados ao sono também podem fazer uso de exercícios regulares, como intervenção não farmacológica, para melhorar o seu ciclo de sono/vigília.Entretanto, devido à quantidade estudos analisados, não é possível afirmar qual o tipo de treinamento é mais eficaz para a melhora da qualidade deste ciclo, se o aeróbico ou o anaeróbico. Outro resultado importante apresentado pela literatura, é que quanto mais sedentário o indivíduo for, está mais propenso a apresentar uma qualidade ruim de sono ou 
distúrbio no ciclo do sono. É importante ressaltar que por mais que esses trabalhos sugiram que a atividade física melhora a qualidade do sono direta ou indiretamente, ainda se faz necessário que mais estudos abordem a temática em questão.

\section{REFERÊNCIAS}

AVIDAN AY, NEUBAUER DN. Chronic Insomnia Disorder. Continuum (Minneap Minn).2017.

BUELA G. Avaliação dos distúrbios de iniciar e manter o sono. In: Rei-mão R. Sono:aspectosatuais.RiodeJaneiro:Atheneu Editora, 1990:33.

CAMPOS, Raquel Munhoz da Silveira et al .Fibromialgia: nível de atividade física equalidade do sono. Motriz: rev. educ. fis. (Online),Rio Claro ,v. 17, n. 3, p. 468-476,Sept.2011.

CARTER, Philippa J et all. Análise longitudinal do sono em relação ao IMC e gordura corporal em crianças: o estudo FLAME. BMJ 2011; 342:d2712

GEIB, L.T.C. et al. Sono e envelhecimento. Rev.psiquiatr. Rio Gd. Sul, v.25, n.3, p.453-465, dez, 2003.

GIL, Antonio Carlos. Como elaborar projetos de pesquisa. 4ed. São Paulo: Atlas, 2007.

LINS-FILHO OL, Queiroz GKF, Santos JFJ, Santos MAM, Oliveira LMFT, Farah BQ.Efeitos do Pilates na qualidade do sono em adultos e idosos: umarevisão sistemática. Rev. Bras. Ativ. Fis. Saúde. 2019;

MARTINSP.J.; MELLO M. T.; TUFIK S. Exercício e sono.Revista Brasileira de Medicina do Esporte,v. 7,n.1, 2001.

MCCARLEY RW. Neurobiology of REM and NREM sleep. Sleep Med 2007; 8: 302-30
MELLO, MARCO T. et al.O exercício físico e os aspectos psicobiológicos.Revista Brasileira de Medicina do Esporte,v.11,n.3, 2005.

PEREIRA,Thúlio;BRASILEIRO-SANTOS, Maria; LIMA, Anna. O treinamento aeróbio melhora o sono dos estudantes universitários?ConScientiaeSaúde, 2018.

PORTELA, L.P.R.; SILVA, L.C. RODRIGUES; OLIVEIRA, L.M.N.; MAZULLO FILHO, J.B.R. Análise da qualidade do sono em mulheres climatéricas ativas e sedentárias. Con Scientiae Saúde, 2017

RÖPKE, Lucilene Maria. Avaliação do efeito de um programa de exercícios do método Pilates na qualidade do sono e na qualidade de vida de pacientes com SAOS, em uso adequado de CPAP. 2017.Acessoem:13demaiode2021.

SANTIAGO, et al. Efeito de uma sessão de treinamento de força sobre a qualidade do sono de adolescentes. Rev. Bras. Med. Esporte- Vol.21,No2-Mar/Abr,2015.

SILVA, Marcia Regina da et al. Sintomas depressivos em idosos e sua relação comdor crônica, doenças crônicas, qualidade do sono e nível de atividade física. BrJP ,SãoPaulo, v.1,n. 4,pág.293-298, dezembro de 2018 .

STEFFENS, Ricardo de Azevedo Klumb et al. Praticar caminhada melhora a qualidade do sono e os estados de humor emmulheres com síndrome da fibromialgia. Rev. dor, São Paulo, v. 12, n. 4, p.327-331,Dec.2011

ZANUTO, Everton Alex Carvalho; CHRISTOFARO, Diego Giulliano Destro; FERNANDES, Rômulo Araújo. Sleep quality and its associations with leisure-time exercise and excess weight among civil servants. Rev. bras. Cineantropom. desempenho hum., Florianópolis, v.16,n. 1, p. 27-35, 2014. 